\title{
Physics Based Algorithms for Sparse Graph Visualization
}

\author{
Milovan Šuvakov \\ Department for Theoretical Physics, Jožef Stefan Institute, Box 3000, 1001 Ljubljana, \\ Slovenia \\ Milovan.Suvakov@ijs.si \\ http://www-f1.ijs.si/ suvakov/
}

\begin{abstract}
Graph visualization represents an important computational tool in analysis of complex networks. Recently, variety of network structures in complex dynamical systems have been found which require appropriately adjusted visualization algorithms. We are testing quantitatively performance of two visualization algorithms based on energy minimization principle on variety of complex networks from cell-aggregated planar graphs to highly clustered scale-free networks. We found that fairly large structures with high clustering can be efficiently visualized with spring energy model with truncated interaction.
\end{abstract}

Keywords: graph layout, complex networks, energy minimization.

\section{Introduction}

Networks which represent complex dynamical systems in physical, biological and social systems usually appear to have strongly inhomogeneous structures [1, which require improved methods for analysis and visualization. Some unusual properties of these networks which may effect visualization procedure are that networks are: sparsely connected, large, strongly inhomogeneous, and/or modular. In addition, constraints such as planarity, absence of clustering, on one side, or small-worldness, scale-free organization, strong clustering, on the other are often making difficulties to the standard visualization procedure [2].

Recent examples include physical models such as: the spring algorithm [3], where the links are replaced by springs with unit natural length and the nodes are interacting with a repulsive force, and Kamada-Kawai algorithm [4, where one looks for the optimal potential that makes the distances between the nodes tend to their corresponding topological distances. Another important ingredient in the visualization algorithm is the actual minimization procedure. Here we studied these two "energy" models 354 and implementing two different algorithms for finding the energy minimum: time integration and metropolis-based algorithm. We compare the efficiency of these two algorithms and analyze the emergent structures for different kinds of networks: trees, scale-free graphs, cellular networks which are planar graphs introduced in [5], and gene interaction network [7. We also suggest modification of the spring algorithm with a truncated interaction and demonstrate it efficiency on these networks. 
Terminology and Definition of the Problem: A graph $G$ is an ordered pair $(V, E)$ given with nonempty set of verteces $V$ and set of edges $E$. If for each edge $(u, v) \in E$ there is an edge $(v, u) \in E$ graph is undirected (symmetrical). The graph visualization is mapping between the verteces and two-dimensional or three-dimensional coordinates $f: V \rightarrow \mathbb{R}^{n}$ (where $n \in\{2,3\}$ ). The quality of mapping is not well defined, but several criteria are generally accepted. One example is minimization of number of edge crossing. While some graphs cannot be drawn without edge crossings, some graphs can. Planar graphs belong to this group. To test uncrossing property of the visualization algorithms in this paper we will use model of cell-aggregated planar graphs which we were presented in 5. Other criteria for satisfactory of visualization often used are: minimization of variance in the edge lengths, maximization of minimal angle between neighboring edges, minimization of the number of bends in the edges, minimization of different slopes used in edge visualization, and other.

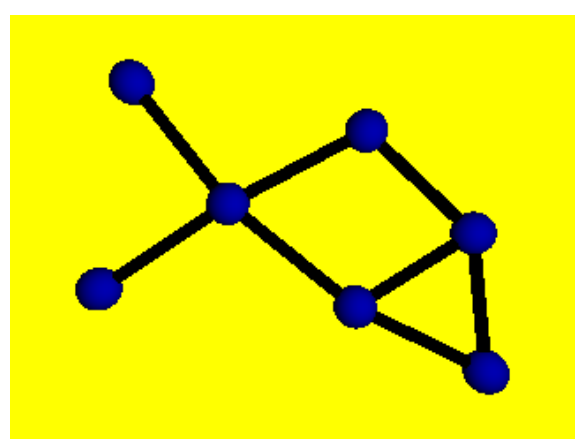

Kamada-Kawai

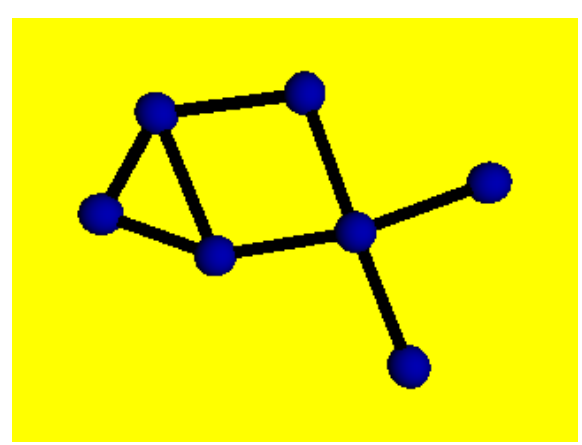

Spring

Fig. 1. Minimal energy configuration in the case of a small graph

In this paper we will concentrate on two-dimensional (planar) visualization of undirected graphs. Also, we will focused on physical models with given Hamiltonian (energy) function which depends on coordinates of vertices and graph topology given by the adjacency matrix. The main idea of this approach is that final planar embedding is given by the minimum of the energy function. The two different "energy" models will be studied supplemented by two different energy minimization algorithms.

\section{2 "Energy" Models and Minimization Algorithms}

The first model is known as Kamada-Kawai 4 model and it is based on idea to place the vertices on such places in the plane with geometric distances correlated with topological distances. The energy in this model is given with:

$$
E=\sum_{i, j} k_{i j}\left(\left|\mathbf{r}_{i}-\mathbf{r}_{j}\right|-l_{i j}\right)^{2},
$$



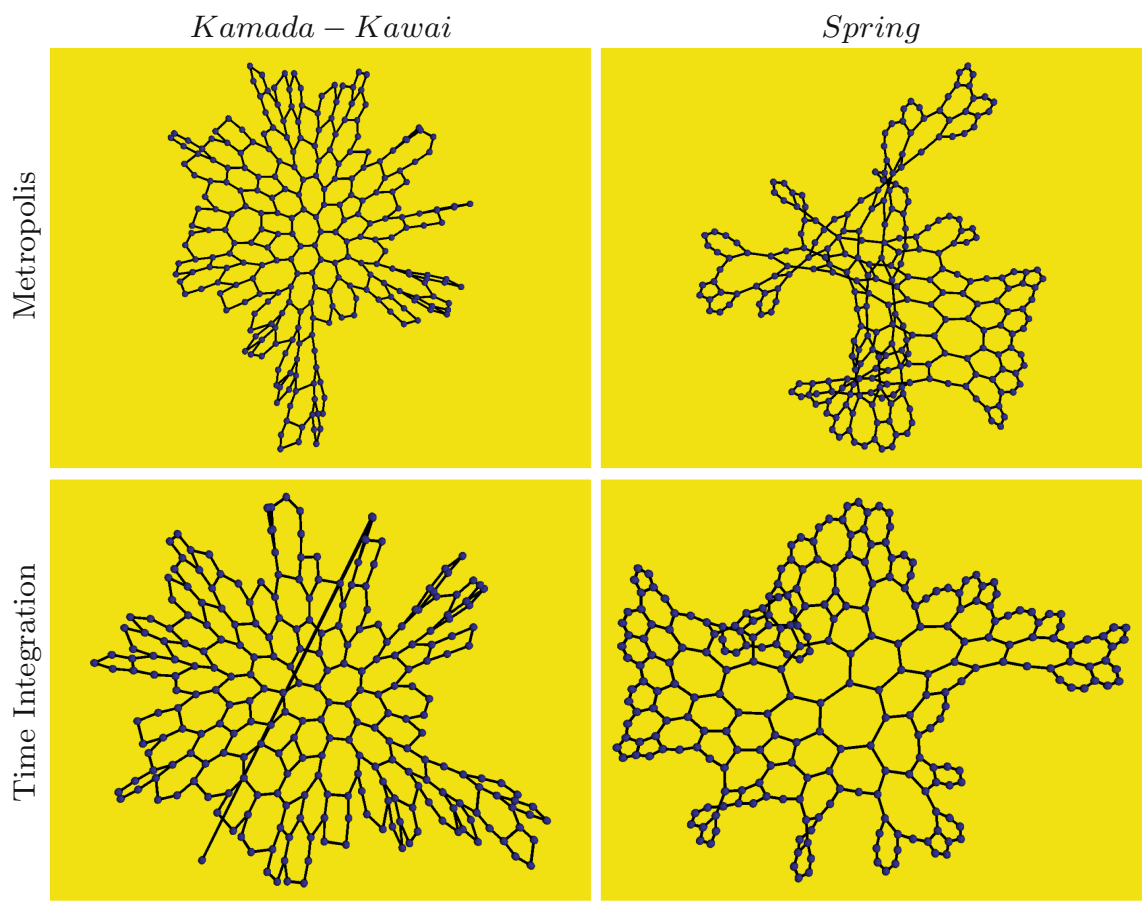

Fig. 2. Same cellular planar graph [5] of $N=256$ nodes shown with four different visualization methods. Relative numbers of edge crossings $\chi$ are: $0.01 \%, 0.31 \%, 0.05 \%$, $0.06 \%$.

where $\mathbf{r}_{i}$ are vectors of positions of vertices, $k_{i j}$ are parameters (in algorithm used in this paper with constant value $k_{i j}=1$ ), and $l_{i j}$ is topological distance between vertices - shortest path length. Shortest path between two vertices is defined as path between them with smallest number of edges 9. In this case, the value of $l_{i j}$ is an positive integer number. The physical interpretation is an harmonic interaction between every two vertices with a minimum on the distance given with shortest path length.

Second model is known as spring model [3] in which the energy is given by:

$$
E=\sum_{(i, j) \in E} k_{i j}\left(\left|\mathbf{r}_{i}-\mathbf{r}_{j}\right|-l_{0}\right)^{2}+\sum_{i, j} \frac{g}{\left|\mathbf{r}_{i}-\mathbf{r}_{j}\right|^{\eta}},
$$

where $k_{i j}, l_{0}, g$ and $\eta$ are parameters, and $\mathbf{r}_{i}$ are vectors of the positions of vertices. In the physical interpretation, first term represent springs with length $l_{0}$ between neighboring verteces and second term represent "electrostatic repulsion" each pair of verteces. 
The illustration of global energy minima for a small graph for both models is given on Fig. 1] For larger graph sizes there are lot of local minima in the energy function, which make the visualization procedure increasingly complex.
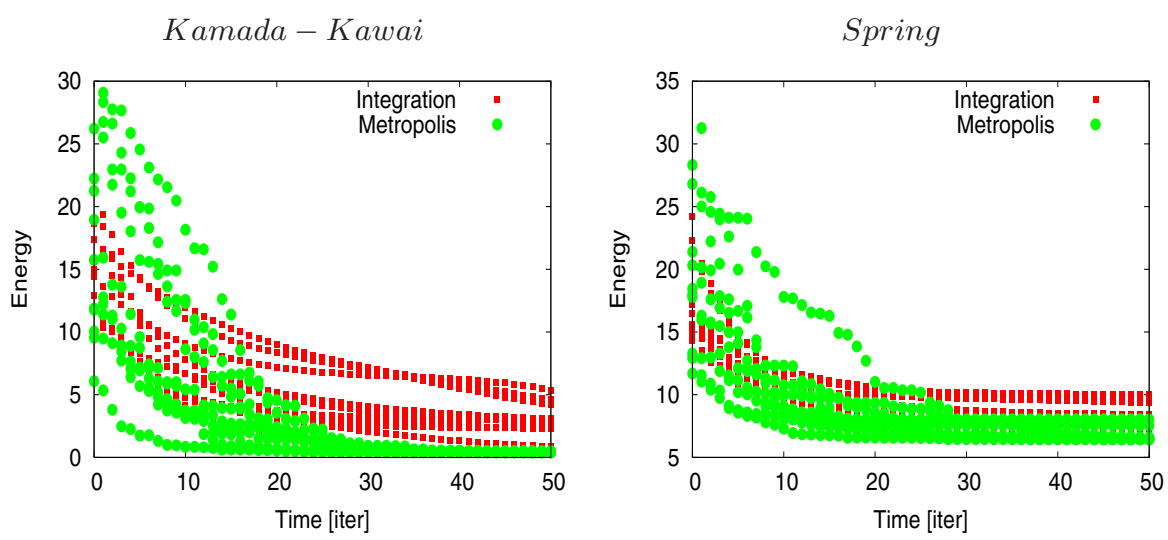

Fig. 3. Relaxation of two energy models for the same planar graph shown on Fig. 2 ant two minimization algorithms. Different lines correspond to different initial conditions.

We show two models for graph visualization given by energy as function of the positions of the vertices. The final planar embedding is given as a minimum of the energy function. In order to find the energy minimum we use two different algorithms: metropolis and time-integration algorithm.

In the metropolis algorithm we calculate the energy change for node random movement, usually taken from Gaussian distribution. The movement is accepted with a probability given by $p=\max \left(1, e^{-\Delta E / T}\right)$, where $\Delta E$ is energy change and $T$ is "temperature" parameter. We implement this algorithm according to following steps:

Initial node positions: randomly given

Do:

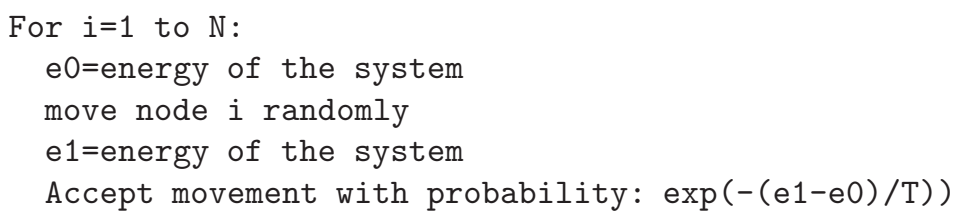

End loop i

Until maximal movement is smaller than a threshold value

As the second algorithm we use the numerical time-integration of the following set of equations:

$$
\frac{\mathrm{d} \mathbf{r}_{i}}{\mathrm{~d} t}=-\nu \frac{\partial E}{\partial \mathbf{r}_{i}},
$$

where $\nu$ is a "viscosity" parameter. It is implemented as follows: 
Initial node positions: randomly given

Do:

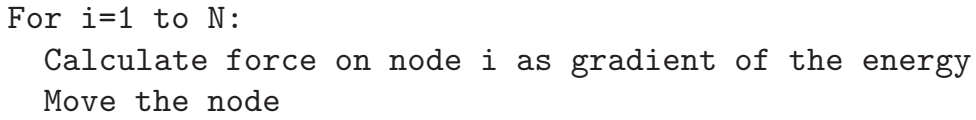

Until maximal movement is smaller than a threshold value
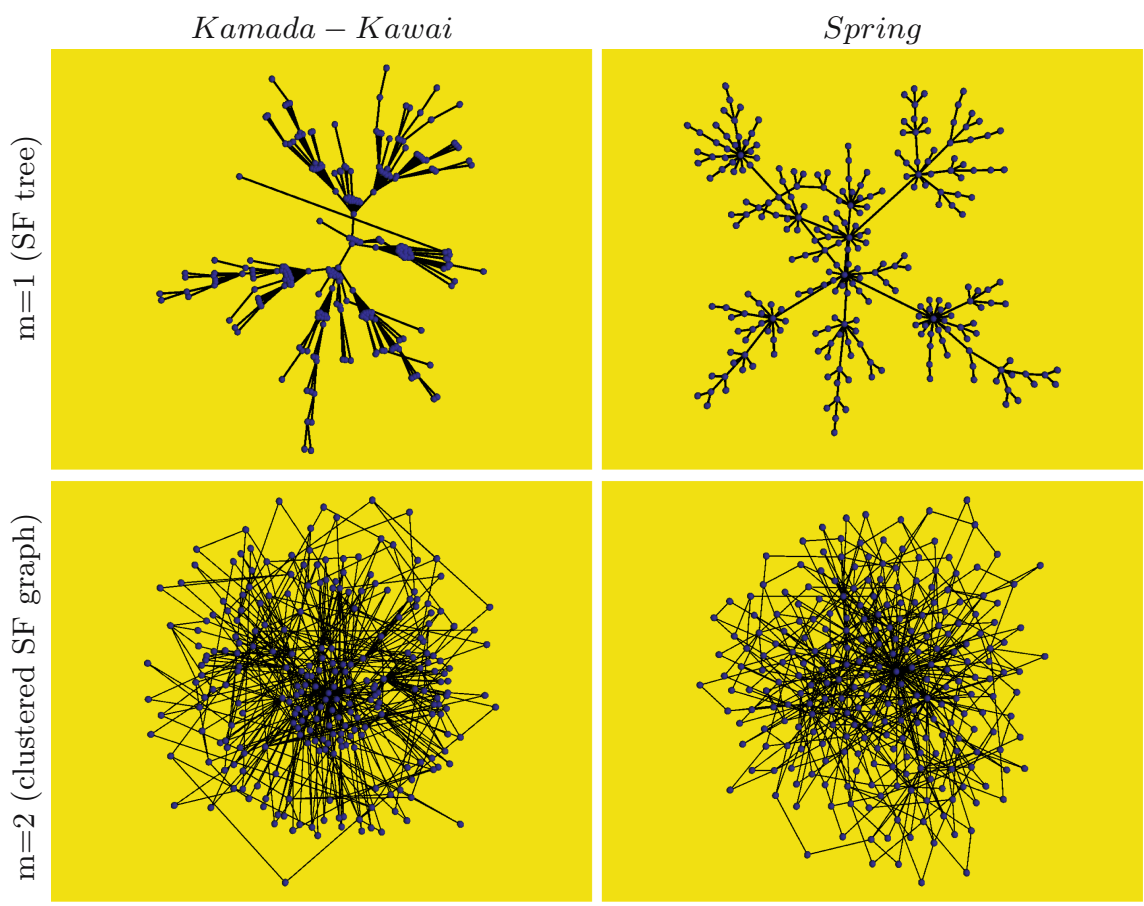

Fig. 4. Emergent layouts for two different models (left column: Kamada-Kawai, right column: spring model) for scale-free tree (top panels), and clustered scale-free graph [6] (bottom panel). In all cases the metropolis minimization algorithm was used. Relative numbers of edge crossings $\chi$ are: $0.70 \%, 0.25 \%, 23.5 \%, 17.0 \%$.

\section{Algorithm Efficiency and the Graph Layouts}

On Fig. 2 we show different graph layouts for the same planar graph with cellular structure [5]. The graph is grown using algorithm given in [5] with control parameters $\mu_{2}=1.0$ and $\nu=1.0$. For reasons of clarity here we use the small size of network is $N=256$. To compare results we will use relative number of 
edge crossings defined as $\chi=N_{c s} / N_{c s, 0}$ where $N_{c s}$ is number of edge crossings for given layout and $N_{c s, 0}$ is average number of edge crossings for the same graph for a random layout. In all results we calculated $\chi$ using value for $N_{c s, 0}$ averaged over 10 random layouts. The Kamada-Kawai method gives better result (see left column on Fig. 21). Quantitatively this difference can be related to faster energy relaxation and absence of many minima shown on Fig. 3. In both cases there are a lot of minima, but in the Kamada-Kawai case a global minima is always found with metropolis algorithm. For the planar graph the difference in the minimization procedure is less pronounced in the case of the spring algorithm.

The comparison between two energy models for scale-free graphs is shown on Fig. 4. In both cases spring algorithm gives better results.

(a)
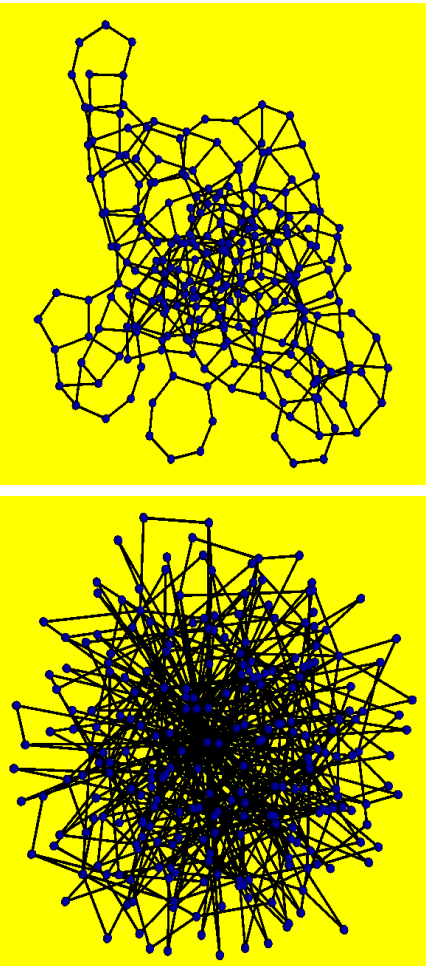

(c) (b)
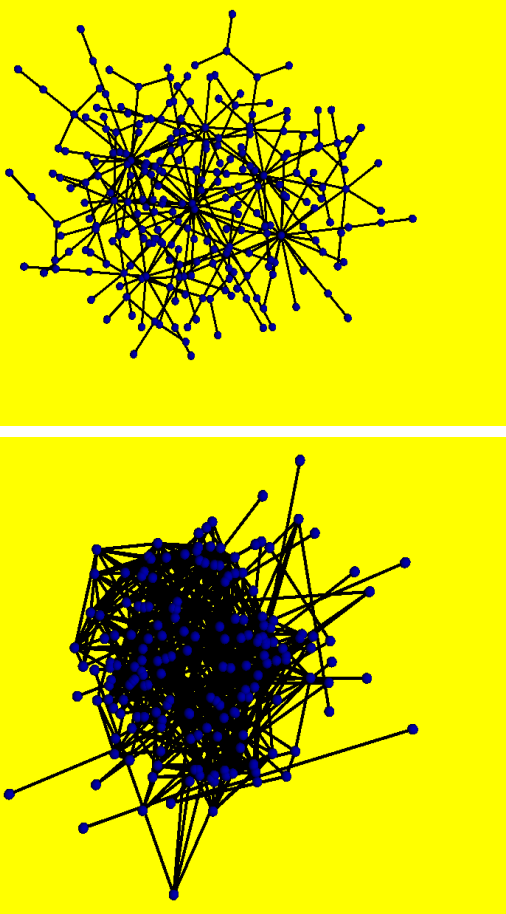

$(d)$

Fig. 5. Emergent layouts in the case of next-neighbor spring model approximation for different graphs: (a) planar graph of Fig. 2. (b) scale-free tree of Fig. 4. (c) clustered scale-free graph of Fig. 4. (d) gene network from Ref. 7]. Relative numbers of edge crossings $\chi$ are: $3.7 \%, 6.0 \%, 25.6 \%, 45.0 \%$. 


\section{Spring Model with Next-Neighbor Interaction}

A considerable computational acceleration in the algorithm is possible if the range of interactions in Eqs. 1 and 2 can be restricted. For instance here we use next-neighbor interactions in the sums in Eqs. 1 and 2 and apply to different graphs. We studied when this approximation gives satisfactory results.

For this purpose we studied the next-neighbor spring model on four different networks shown on Fig. 5. planar cellular graph, scale-free tree, clustered sparse scale-free graph, and gene network.

(a)

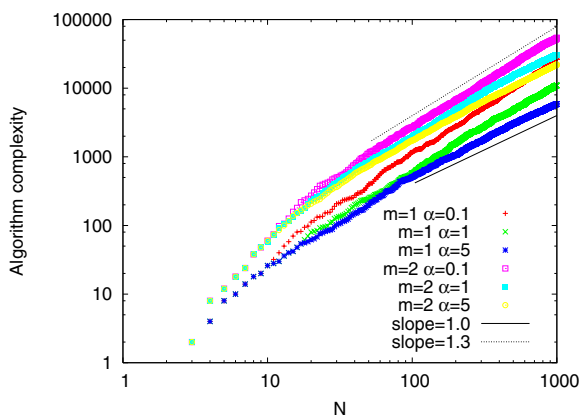

(b)

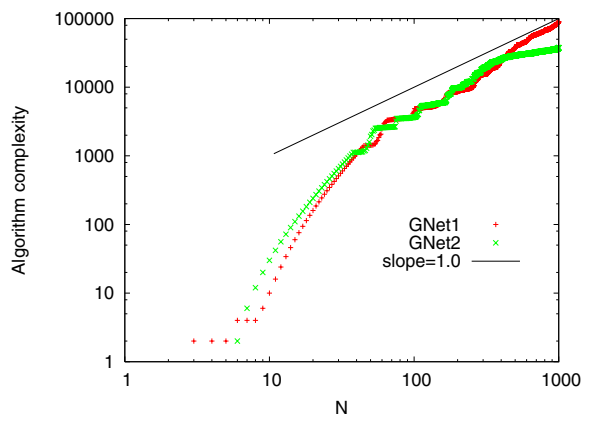

Fig. 6. Algorithm complexity $N \times<k_{n n}>$ on size of network $N$ : (a) Growing network model from [6] for different model parameters (b) Gene networks from [7]

In the case when clustering is too small the emergent layouts are not well outstretched. However, in the case of sparse networks with higher clustering coefficient the algorithm seems much better (see Fig. 5(c)). In the case of notscalefree gene network, see example in Fig. 5 (d) the next-neighbor approximation does not give a good layout.

The complexity of the energy calculation algorithm is proportional to the mean value of next-neighbors $<k_{n n}>$. The one computational step (loop through all nodes) in both energy minimization algorithms takes $N \times<k_{n n}>$ calculations. Since the mean value $\left\langle k_{n n}>\right.$ increases with system size and saturates at a threshold, the complexity of energy calculation algorithm for all nodes appears to be proportional to system size $N$ instead to $N^{2}$. For the network sizes around the threshold value, the exponent is between one and two (see Fig. 6).

\section{Conclusions and Future Work}

We tested three energy models for two-dimensional visualization of graphs with given size and structure defined with adjacency matrix $C_{i j}$. We give certain quantitative measures of efficiency of the energy minimization algorithm. We 
Table 1. For different graph structures the listed are methods of choice with metropolis minimization

\begin{tabular}{llr}
\hline \hline Graph structure & Visualization Method of Choice & $\chi$ \\
\hline \hline Planar graph with loops & Kamada-Kawai model & $0.01 \%$ \\
\hline Scale-free trees & Spring model - infinite range interaction & $0.26 \%$ \\
\hline Scale-free clustered & Spring model- short range interaction & $26 \%$ \\
\hline Non-SF clustered & $? ?$ & $45 \%$ \\
\hline \hline
\end{tabular}

presented layouts for different type of graphs, in particular the graphs which appear in applications of statistical physics to real-world networks. In summary we find that different algorithms work differently depending on the graph structure. For particular graph type we summarize the method of choice in Table 1 .

Another idea for visualization of complex networks is to use growth models, starting with small sub-graph and minimizing the energy while adding nodes one by one with known patterns of links (see online java applet on author's web page [12]).

Acknowledgments. M.S. thanks financial support from the Marie Curie Research and Training Network MRTN-CT-2004-005728 project. Many thanks to my $\mathrm{PhD}$ supervisor prof. Bosiljka Tadić for her useful help and guidance.

\section{References}

1. Boccaletti, S., Latora, V., Moreno, Y., Chavez, M., Hwang, D.U.: Complex networks: Structure and dynamics. Physics Reports 424, 175-308 (2006)

2. Batagelj, V., Mrvar, A.: Pajek, http://vlado.fmf.uni-lj.si/pub/networks/ pajek/

3. Eades, P.: A heuristic for graph drawing. Congraessus Numerantium 42, 149-160 (1984)

4. Kamada, T., Kawai, S.: An algorithm for drawing general undirected graphs. Inf. Process. Lett. 31, 7-15 (1989)

5. Šuvakov, M., Tadić, B.: Topology of Cell-Aggregated Planar Graphs. In: Alexandrov, V.N., van Albada, G.D., Sloot, P.M.A., Dongarra, J. (eds.) ICCS 2006. LNCS, vol. 3993, pp. 1098-1105. Springer, Heidelberg (2006)

6. Tadić, B.: Dynamics of directed graphs: the world-wide Web. Physica A 293, 273 (2001)

7. Živković, J., Tadić, B., Wick, N., Thurner, S.: Statistical indicators of collective behavior and functional clusters in gene networks of yeast. The European Physical Journal B - Condensed Matter and Complex Systems 50(1), 255-258 (2006)

8. Dorogovtsev, S.N., Mendes, J.F.F.: Evolution of Networks. Oxford University Press, Oxford (2003)

9. Bollobás, B.: Modern Graph Theory. Springer, New York (1998)

10. Tadić, B.: From Microscopic Rules to Emergent Cooperativity in Large-Scale Patterns. In: Krasnogor, N., Gustafson, S., Pelta, D., Verdegay, J.L. (eds.) Systems Self-Assembly: multidisciplinary snapshots, Elsevier, Amsterdam (2005)

11. Ahuja, R.K., Magnanti, T.L., Orlin, J.B.: Network Flows: Theory, Algorithms, and Applications. Prentice-Hall, New Jersey (1993)

12. Suvakov M.: http://www-f1.ijs.si/ suvakov/ 